\title{
Intracavity trace molecular detection with a broadband mid-IR frequency comb source
}

\author{
Magnus W. Haakestad, ${ }^{1,2}$ Tobias P. Lamour, ${ }^{1,3}$ Nick Leindecker, ${ }^{1}$ \\ Alireza Marandi, ${ }^{1}$ and Konstantin L. Vodopyanov ${ }^{1,4, *}$ \\ ${ }^{1}$ E. L. Ginzton Laboratory, Stanford University, Stanford, CA 94305, USA \\ ${ }^{2}$ Norwegian Defence Research Establishment (FFI), P O Box 25, \\ NO-2027 Kjeller, Norway \\ ${ }^{3}$ Scottish Universities Physics Alliance (SUPA), \\ Institute of Photonics and Quantum Sciences, School of Engineering and Physical Sciences, \\ Heriot-Watt University, Riccarton, Edinburgh EH14 4AS, UK \\ ${ }^{4}$ Univ. Central Florida, CREOL, College of Optics \& Photonics, Orlando, FL 32816, USA \\ *Corresponding author: vodopyan@stanford.edu
}

\begin{abstract}
Ultrasensitive detection of methane, isotopic carbon dioxide, carbon monoxide, formaldehyde, acetylene and ethylene is performed in the spectral range $2.5-5 \mu \mathrm{m}$ using intracavity spectroscopy in broadband optical parametric oscillators (OPOs). The OPOs were operated near degeneracy and synchronously pumped either by a mode-locked erbium (1560 nm) or thulium (2050 nm) fiber laser. A large instantaneous bandwidth of up to $800 \mathrm{~cm}^{-1}$ allows for simultaneous detection of several gases. We observe an effective path length enhancement due to coherent interaction inside the OPO cavity and achieve part-per-billion sensitivity levels. The measured spectral shapes are in good agreement with a model that takes into account group delay dispersion across the broad OPO frequency band. (C) 2018 Optical Society of America
\end{abstract}

OCIS codes: 010.1030, 120.3940, 190.4410, 300.6340, 320.7110. 


\section{Introduction}

Many important gases strongly absorb in the mid-IR $(>2.5 \mu \mathrm{m})$ 'signature' region due to their fundamental rotational-vibrational transitions in this wavelength range. Optical spectroscopy in the mid-IR region has potential for such applications as trace gas detection [1], remote chemical sensing [2], and human breath analysis [3 5]. For example, human breath is known to contain more than 500 different 'biomarker' volatile organic compounds and quantification of these gases may have clinical applications.

Simultaneous detection of several gases requires a suitable broadband or a widely tunable CW source. Compared to narrow bandwidth sources, a broadband source coupled with Fourier transform methods offers advantages for spectroscopy including massive parallelism of data collection and elimination of the need for wavelength tuning. Optical frequency combs are particularly attractive broadband sources for spectroscopy [6], owing to their extraordinary coherence over broad bandwidth. This property has led to applications including trace gas detection, molecular fingerprinting and dual comb spectroscopy [7-10]. Even though the resolution needed for gas spectroscopy may be much lower than the line spacing of frequency combs, one can utilize the comb structure to improve the sensitivity of trace gas detection. This can be done by coupling the frequency comb into a high-finesse Fabry-Perot cavity [11-13], where effective path lengths of several km can be obtained [14].

Synchronously pumped (sync-pumped) optical parametric oscillators represent an attractive way of generating mid-IR frequency combs suitable for molecular spectroscopy [14 17]. Recently, our group implemented a new method for generating broadband mid-IR combs, based on a doubly resonant, degenerate sync-pumped OPO, which rigorously downconverts and augments the spectrum of its pump frequency comb [18,19]. Exceptionally large parametric gain bandwidth at degeneracy combined with extensive cross mixing of comb components, resulted in extremely broad ( $>$ one octave) instantaneous mid-IR bandwidth extending the wavelength range beyond $6 \mu \mathrm{m}$ [19]. Here we show that such a broadband source, combined with intracavity spectroscopy, becomes a powerful tool for trace molecular detection.

We perform molecular spectroscopy using two such sources. One source is a periodicallypoled lithium niobate (PPLN) based OPO, pumped at $1.56 \mu \mathrm{m}$ by a femtosecond Er-doped fiber laser [18, and the other source is an orientation-patterned GaAs based OPO, pumped at $2.05 \mu \mathrm{m}$ by a Tm-doped fiber laser [19]. Both OPOs operate near degeneracy (central wavelength is twice that of the pump) - to obtain broad instantaneous bandwidth. In this work the OPO cavity itself is used as an enhancement cavity to increase the effective path length [20 22]. Intracavity spectroscopy of methane, carbon monoxide, formaldehyde and several other gases is performed by injecting gas directly into the OPO enclosure, or by using an intracavity gas cell with Brewster windows. We observe significant effective path length enhancement due to the intracavity action. In addition, we find that the measured spectral 
line shapes may have dispersive features. Such features have previously been observed with cavity-enhanced frequency comb spectroscopy [14,23], and in intracavity spectroscopy with sync-pumped OPOs and mode-locked lasers [19,24,25]. The measured spectra are compared to a simple model, based on the intracavity passive loss and round-trip dispersion, and excellent agreement between theory and measurements is found.

The remainder of the paper is organized as follows. In Sec. 2, we introduce a theory on how intracavity dispersion affects the spectrum and phases of the OPO comb lines. Section 3 presents a simple theory of intracavity spectroscopy with broadband OPOs. In Secs. 4 and 5 we describe the experimental setup and measurement procedures. Sections [6 and 7 describe correspondingly results with the Er- and Tm-pumped systems. Finally, in Sec. 8, we discuss the results and make conclusions in Sec. 9 .

\section{Intracavity dispersion and OPO comb width}

In the case of femtosecond pumping by a mode-locked laser, the pump and the OPO output are represented by a manifold of comb lines sharing the same spacing, equal to the pump pulse repetition frequency $f_{\text {rep }}$. The pump field is represented by a comb of frequencies $\nu_{p, n}=f_{\mathrm{CEO}, p}+n f_{\mathrm{rep}}$, while the OPO idler and signal follow $\nu_{i, l}=f_{\mathrm{CEO}, i}+l f_{\mathrm{rep}}$ and $\nu_{s, m}=$ $f_{\mathrm{CEO}, s}+m f_{\mathrm{rep}}$, correspondingly. Here $l, m$, and $n$ are integers, and $f_{\mathrm{CEO}}$ is the carrier-envelope offset frequency $\left(0 \leq f_{\mathrm{CEO}}<f_{\text {rep }}\right)$.

From photon energy conservation it follows that for integers $l$ and $m$, there should be an integer $n$, such that

$$
\nu_{i, l}+\nu_{s, m}=\nu_{p, n},
$$

from which it follows that there are two solutions for $f_{\mathrm{CEO}}$ of the signal and idler:

$$
\begin{aligned}
& f_{\mathrm{CEO}, s}+f_{\mathrm{CEO}, i}=f_{\mathrm{CEO}, p} \\
& f_{\mathrm{CEO}, s}+f_{\mathrm{CEO}, i}=f_{\mathrm{CEO}, p}+f_{\mathrm{rep}}
\end{aligned}
$$

For a degenerate OPO, where the signal and idler become indistinguishable, $f_{\mathrm{CEO}, s}=f_{\mathrm{CEO}, i}$, hence Eq. (2) becomes

$$
\begin{aligned}
& f_{\mathrm{CEO}, s}=\frac{f_{\mathrm{CEO}, p}}{2}, \\
& f_{\mathrm{CEO}, s}=\frac{f_{\mathrm{CEO}, p}}{2}+\frac{f_{\mathrm{rep}}}{2} .
\end{aligned}
$$

In order for a broadband degenerate OPO to oscillate, it is necessary that for each OPO comb frequency, the accumulated phase delay per roundtrip, $\Delta \phi=k L$, is an integer of $2 \pi$ (here $k$ is the average value of the wavevector and $L$ is the roundtrip cavity length). This can be achieved in vacuum $(k=2 \pi \nu / c)$ by choosing $L=c / f_{\text {rep }}$ when $f_{\mathrm{CEO}, p}=0$ ( $c$ corresponds to the speed of light). However due to dispersion in a real cavity, $\Delta \phi$ is no longer linear with 


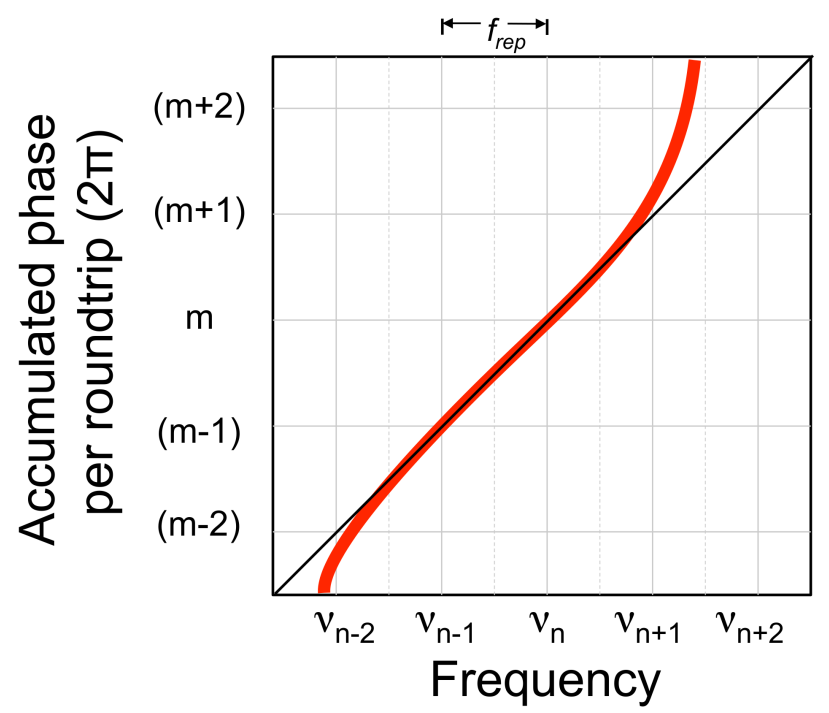

Fig. 1. For a broadband OPO to oscillate, it is necessary that for each comb line, $\nu_{s, m}$, the phase delay $k L$ per roundtrip is an integer multiple of $2 \pi$. Due to dispersion in the cavity, the roundtrip phase (thick line) is no longer a straight line. As a result, peripheral comb lines acquire extra phase, which prevents them from oscillation. Dashed vertical lines correspond to the alternative set of OPO comb lines shifted in frequency by $f_{\text {rep }} / 2$. The frequency spacing between the comb lines is highly exaggerated for clarity.

$\nu$ (Fig. 1, thick line) and peripheral comb teeth acquire extra phase preventing them from oscillation.

Figure 2 plots the calculated extra phase accumulated per roundtrip near the degeneracy of an erbium (1560 nm) laser pumped PPLN OPO, at different cavity lengths near the optimum sync-pumped condition. Here we took into account dispersion originating from (i) $0.5-\mathrm{mm}$ PPLN, (ii) 2-mm ZnSe dispersion compensator, and (iii) dielectric mirror coating. These components have a group delay dispersion of $-287 \mathrm{fs}^{2}, 296 \mathrm{fs}^{2}$, and $35 \mathrm{fs}^{2}$, respectively, at $3.12 \mu \mathrm{m}$. Effectively, Fig. 2 is a residue between the two curves of Fig. 1. When $L$ is decreased (from left to right in Fig. 22), the phase curve rotates clockwise. Broadband OPO operation occurs when the accumulated phase for all the comb lines is an integer multiple of $2 \pi$. Because there is an alternative set of OPO comb lines Eq. (3b), the interval between discrete cavity lengths corresponds to $\pi$ instead of $2 \pi$, in terms of the phase shift (this is expressed by dashed horizontal lines), so that the oscillation peaks are spaced by $\Delta L \approx \lambda_{\text {pump }}[26$. Cases (a)-(c) of Fig. 2 correspond to degenerate OPO operation, with the broadest spectrum expected for (c), while (d) is a non-degenerate (but still doubly resonant) case, when the 


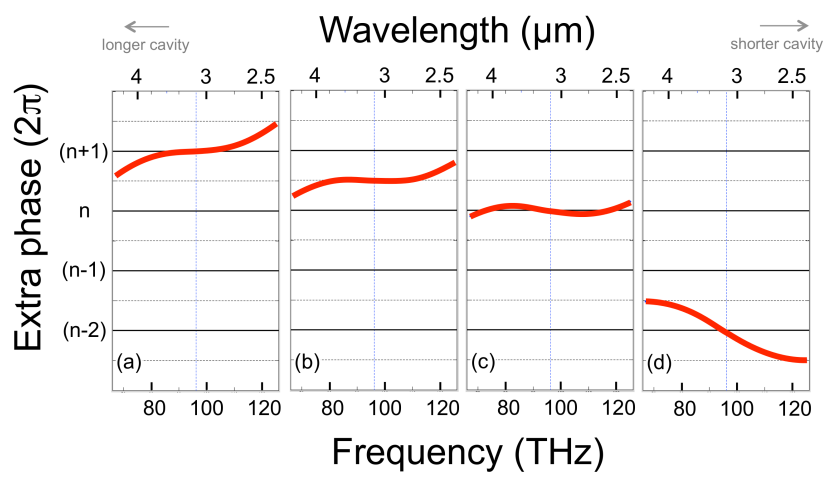

Fig. 2. Calculated extra phase per roundtrip near OPO degeneracy (vertical dotted line, $96 \mathrm{THz}, 3.12 \mu \mathrm{m}$ ) at different cavity roundtrip lengths $L$ near the optimum. The length steps between (a)-(b) and (b)-(c) are $1.56 \mu \mathrm{m}\left(\approx \lambda_{\text {pump }}\right)$. Dispersion originating from a $0.5-\mathrm{mm}$ PPLN, 2-mm ZnSe, and a dielectric mirror was taken into account. The cavity length is decreasing from (a) to (d).

comb lines are clustered around separate signal and idler peaks (at $\sim 70$ and $120 \mathrm{THz}$ ).

Thus, intracavity dispersion is one of the main factors that restrict the OPO bandwidth and can also introduce frequency chirp of the OPO pulses.

\section{Model for intracavity molecular spectroscopy}

Now we consider a high-finesse synchronously pumped femtosecond OPO in a steady-state condition. The round-trip evolution of the electric field $A$ of a resonating OPO comb line at the frequency $\nu_{s, m}$ is given by

$$
A=t A+\Delta A .
$$

Here we assume that $A=A_{s, m} \exp \left(2 \pi i \nu_{s, m} t\right), t=|t| \exp (-i \Delta \phi)$ is the complex round-trip amplitude transmission coefficient of the OPO cavity, $\Delta A$ is the nonlinear optical gain due to the presence of the pump field, and $\Delta \phi$ - is the phase delay acquired per round-trip. The rationale for using the form Eq. (44) is as follows: For a 3-wave interaction $\left(\nu_{s, m}+\nu_{i, l}=\nu_{p, n}\right)$ in the slowly varying envelope approximation, the equation describing the evolution of the OPO field $A\left(\nu_{s, m}\right)$ in a nonlinear crystal is given by $\frac{\mathrm{d}}{\mathrm{d} z} A\left(\nu_{s, m}\right)=i \kappa A\left(\nu_{p, n}\right) A\left(\nu_{i, l}\right)^{*}$, where $\kappa$ is a constant, proportional to the nonlinear susceptibility $\chi^{(2)}$, while assuming perfect phase-matching. In the sync-pumped OPO case, the nonlinear interaction results in a crosscoupling between large manifolds of pump and OPO comb lines (Fig. 3), such that for the $m$-th mode

$$
\frac{\mathrm{d}}{\mathrm{d} z} A\left(\nu_{s, m}\right)=i \kappa \sum_{\nu_{p, n}} \sum_{\nu_{i, l}=\nu_{p, n}-\nu_{s, m}} A\left(\nu_{p, n}\right) A^{*}\left(\nu_{i, l}\right) .
$$




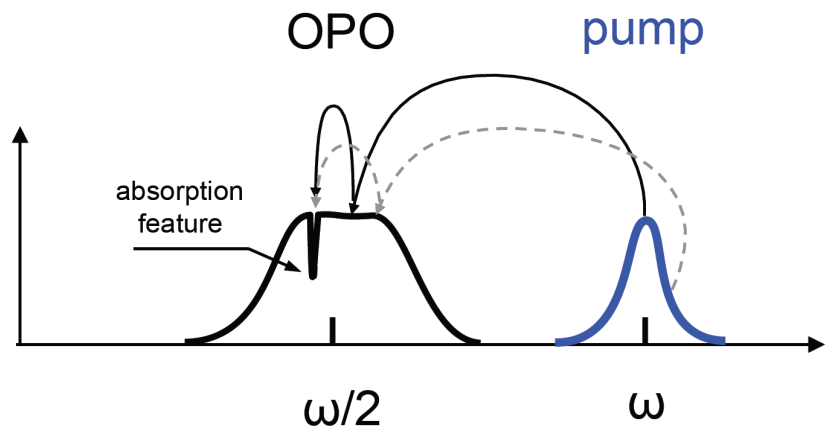

Fig. 3. In a broadband OPO, parametric gain at a given frequency is the result of cross-coupling between large amounts of pump and OPO comb lines. Two such paths are shown.

Here $A\left(\nu_{p, n}\right)$ refers to the pump, $A\left(\nu_{i, l}\right)$ - to the 'complementary' OPO wave, and a star denotes its complex conjugate. Given the extremely broad spectra for both the pump and the OPO, the presence of narrow molecular absorption features in the spectra will be averaged out in Eq. (5). Based on this reasoning, we make the approximation that the right hand side of Eq. (5) is not significantly affected by weak, narrow molecular absorption features, and integration over the crystal length gives the constant field 'gain' $\Delta A$ in Eq. (44).

\section{A. Intracavity absorption enhancement, real amplitude transmission}

Let us first regard a simple case of high-finesse cavity when the OPO comb lines are in exact resonance with the cavity, $\exp (-i \Delta \phi)=1$, and the round-trip amplitude transmission $t(\nu)$ is real: $t_{0}=\exp \left(-\delta_{0}\right)$, where $\delta_{0} \ll 1$ is the round-trip amplitude loss, such that $\delta_{0}=\delta_{o}^{I} / 2$, where $\delta_{0}^{I}$ is the intensity (or power) loss. Also, let us assume that a small real (as in the center of a molecular absorption line) roundtrip absorption $\delta_{1}$ is introduced inside the cavity, such that $\delta_{1} \ll \delta_{0}$, corresponding to the amplitude transmission coefficient $t_{1}=\exp \left(-\delta_{1}\right)$. In this case, Eq. (4) results in

$$
A=\frac{\Delta A}{1-t_{0} t_{1}} \approx \frac{\Delta A}{\delta_{0}+\delta_{1}}=\frac{\Delta A}{\delta_{0}\left(1+\delta_{1} / \delta_{0}\right)} .
$$

The relative field change $\delta A / A$, due to molecular absorption, is $-\delta_{1} /\left(\delta_{0}+\delta_{1}\right) \approx-\delta_{1} / \delta_{0}$, instead of $-\delta_{1}$ if one measures transmission change due to molecular gas outside the OPO cavity. Consequently, the high-finesse OPO cavity 'amplifies' the small absorption by a factor $1 / \delta_{0}$. This can also be expressed in terms of the photon lifetime inside the cavity. The photon number decays in a cavity as $n \sim n_{0} \exp \left(-2 \delta_{0} f_{\text {rep }} t\right)$, and thus $n$ reduces by a factor $1 / e$ after $N=1 /\left(2 \delta_{0}\right)$ cavity roundtrips. Hence the absorption enhancement due to the intracavity 
action, which results in a corresponding increase of the effective path length through the gas, is $2 N$.

\section{B. Intracavity absorption enhancement, complex amplitude transmission}

When an OPO comb line acquires extra phase $\Delta \phi$ per round trip, the transmission $t$ is complex: $t_{0}=\exp \left(-\delta_{0}-i \Delta \phi\right)$. Also, molecular absorption (we assume for simplicity a Lorentzian line shape, which is valid at pressures $>0.1 \mathrm{~atm}$ ), can be characterized close to resonance by a complex transmission function [27]

$$
t_{1}=\exp \left(-\frac{i \delta_{1}}{\left(\nu_{0}-\nu\right) / \gamma+i}\right)
$$

Here $\delta_{1}$ is the amplitude loss per roundtrip at the resonant frequency $\nu_{0}$, and $\gamma$ is the line half width. For a known gas and known path length $L_{\text {mol }}, \delta_{1}$ for a given molecular transition can be found from the HITRAN database through the formula $\delta_{1}=\frac{1}{2} \delta_{1}^{I}=\frac{1}{2} \sigma_{\text {mol }}\left(\nu_{0}\right) n_{\text {mol }} L_{\text {mol }}=$ $\frac{1}{2} \frac{S}{\pi \gamma} n_{\mathrm{mol}} L_{\mathrm{mol}}$, where $S$ is the pressure-independent absorption intensity for a given energy transition, that is absorption cross-section per molecule integrated over the frequency [28], $\sigma_{\text {mol }}\left(\nu_{0}\right)$ is the peak absorption cross-section, and $n_{\text {mol }}$ is molecular concentration. Similar to Eq. (6), we can write an expression for the field amplitude:

$$
A=\frac{\Delta A}{1-t_{0} t_{1}} \approx \frac{\Delta A}{1-\exp \left(-\delta_{0}-i \Delta \phi-\frac{i \delta_{1}}{\left(\nu_{0}-\nu\right) / \gamma+i}\right)}
$$

When $\Delta \phi \neq 0$, derivative-like features appear in the spectrum, as illustrated by Fig. 4 . Physically, dispersion in the OPO cavity introduces a mismatch $\Delta \phi(\nu)$ between the OPO frequency comb and the cavity resonances. Molecular dispersion imposes an additional phase shift near absorption resonances. This causes a change of the spectral line shapes depending on the mismatch.

Accordingly, our simple theory for intracavity spectroscopy with femtosecond OPOs predicts dispersive features in the absorption spectrum, similar to those observed with frequency comb spectroscopy enhanced with an external cavity [23, 29].

\section{Experimental setup}

The first OPO in our experiment was based on a PPLN crystal and was pumped by an Er:fiber laser (Toptica, $350 \mathrm{~mW}$ average power, $1.56 \mu \mathrm{m}$ wavelength, $80 \mathrm{MHz}$ repetition rate, 85 fs pulse duration), producing an output centered at $\sim 3.1 \mu \mathrm{m}$. The length of the PPLN crystal was $0.5 \mathrm{~mm}$ or $0.8 \mathrm{~mm}$ and a ZnSe 1-degree wedge pair was used for dispersion compensation and for out-coupling, as shown in Fig. 5. The second OPO was based on a 0.5-mm-long orientation-patterned GaAs (OP-GaAs) crystal, and was pumped by a Tm:fiber laser (IMRA, $600 \mathrm{~mW}$ average power, $2.05 \mu \mathrm{m}$ wavelength, $75 \mathrm{MHz}$ rep. rate, $93 \mathrm{fs}$ pulse 

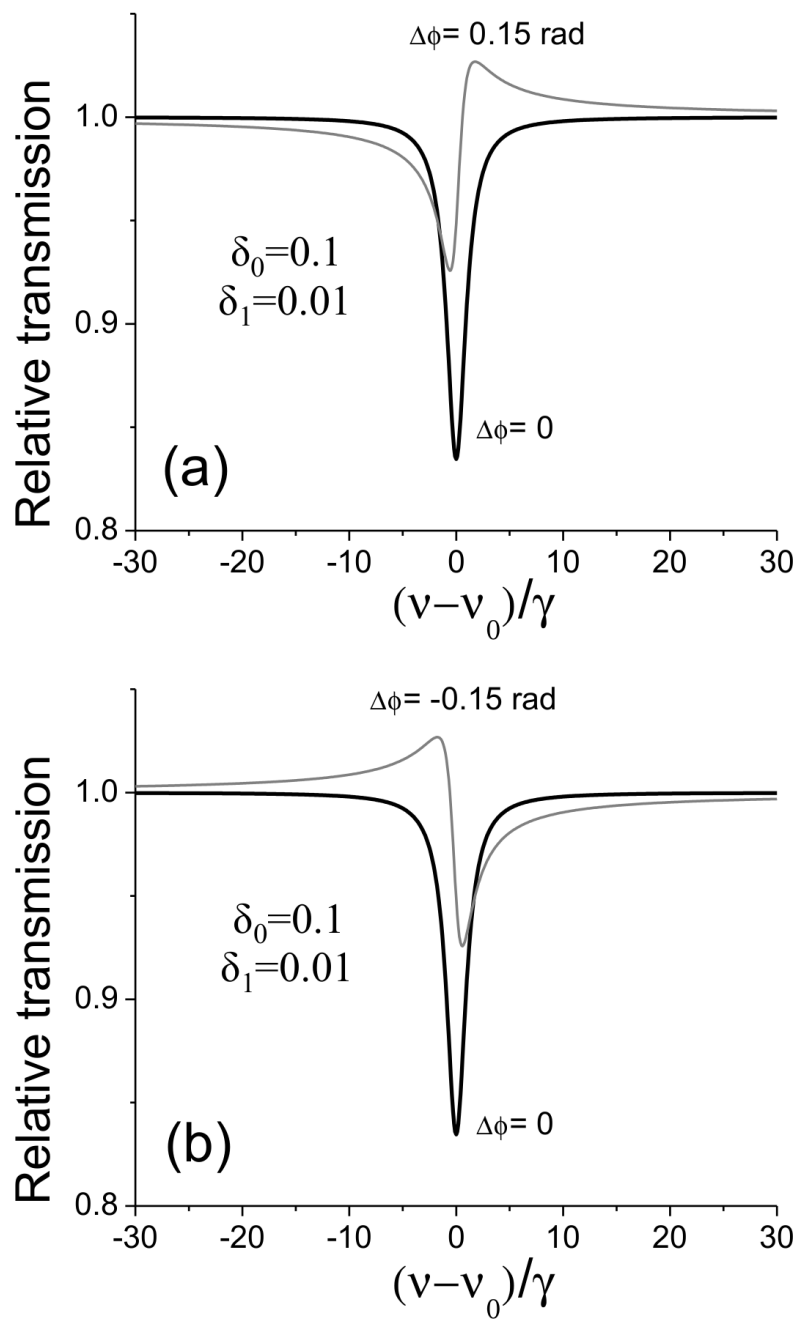

Fig. 4. When the roundtrip phase differs from an integer times $2 \pi$, dispersive features of both signs appear in the molecular spectrum. (a) Calculated relative spectral intensity $|A|^{2}$ near molecular resonance vs. normalized frequency at $\Delta \phi=0$ and $\Delta \phi=0.15$. (b) Same at $\Delta \phi=0$ and $\Delta \phi=-0.15$.

duration), producing an output centered at $4.1 \mu \mathrm{m}$. The OPOs and their coherence properties are described in detail in Refs. [18, 19, 26]. Both OPOs were placed in Plexiglas enclosures and the pump lasers were free running. The output power of the OPOs was some tens of $\mathrm{mW}$.

Oscillation occurs at a discrete set of cavity lengths, separated (in effective roundtrip cavity length) by approximately one pump wavelength, due to the doubly resonant operation [26]. Figure [6] shows measured output power of the Er:fiber pumped OPO, with $0.8 \mathrm{~mm}$ PPLN, as a function of cavity length detuning. The cavity length was locked to one of these oscillation 


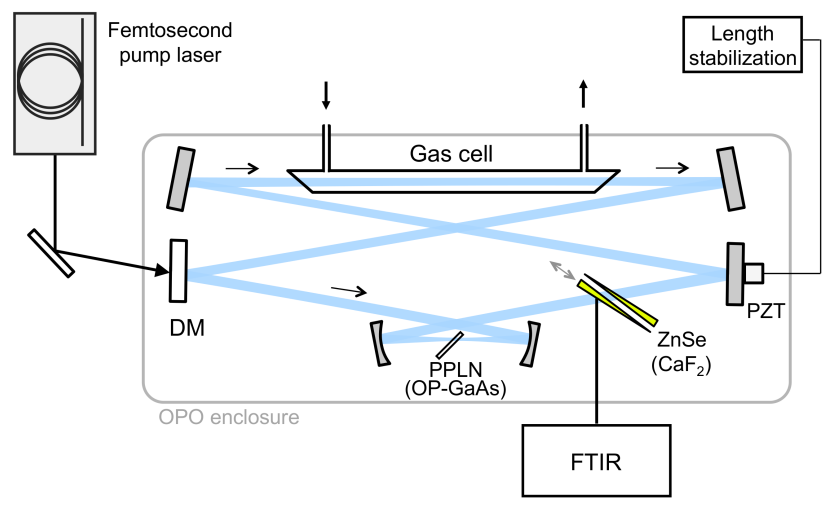

Fig. 5. Schematic of the degenerate broadband OPO. The pump beam was introduced through the in-coupling dielectric mirror DM. The other five mirrors are gold coated. A pair of wedges made of ZnSe (Er:fiber-pumped system) or $\mathrm{CaF}_{2}$ (Tm:fiber-pumped system) was used for (i) dispersion compensation and (ii) beam out-coupling. The nonlinear crystal was AR-coated (PPLN) or placed at Brewster's angle (OP-GaAs).

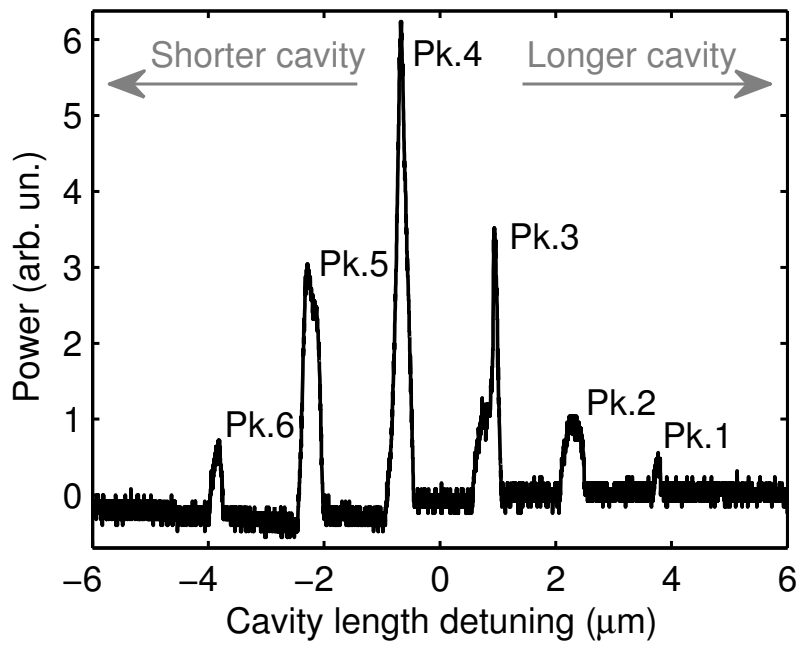

Fig. 6. Output power of the Er:fiber pumped OPO, with $0.8 \mathrm{~mm}$ PPLN, as a function of cavity length (roundtrip) detuning.

peaks using a feedback loop including a piezo stage attached to one of the cavity mirrors. Figure 7 shows measured spectra for the Er:fiber OPO for a selection of oscillation peaks, with $\mathrm{N}_{2}$-purging of the OPO enclosure. The spectra are shown for the two different PPLN lengths used in the experiments. We observe that the maximum spectral width is limited 


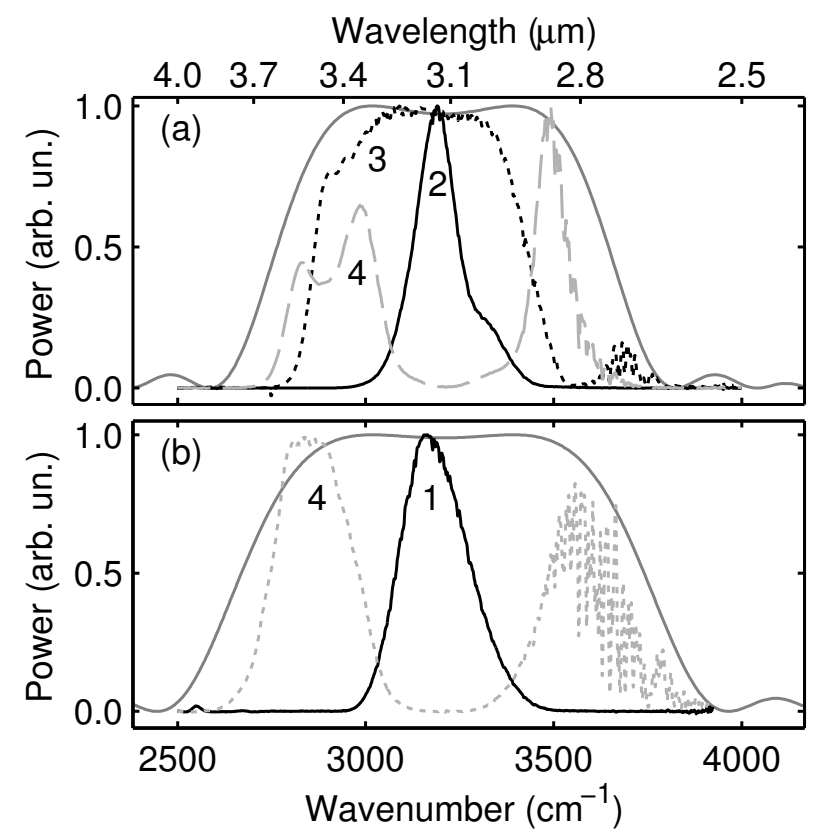

Fig. 7. Measured output spectra for the Er:fiber pumped OPO using (a) 0.8 $\mathrm{mm}$ PPLN and (b) $0.5 \mathrm{~mm}$ PPLN. The spectra are shown for oscillation peaks No. 2-4 in (a) and peaks No. 1 and 4 in (b). Also shown (solid gray lines) are the theoretical PPLN parametric gain spectra for the two crystal lengths.

by the parametric gain bandwidth of the nonlinear PPLN crystal. The shape of the OPO spectra is strongly dependent on (i) intracavity dispersion and (ii) on which oscillation peak the OPO is locked to [19]. Figure 8 shows measured spectrum for one of the oscillation peaks for Tm:fiber pumped OPO.

\section{Measurement}

We have performed spectroscopic measurements of six different molecules. Measurements of methane, formaldehyde, ethylene and acetylene were performed using the Er:fiber pumped $\mathrm{OPO}$, while the absorption spectra of carbon monoxide and isotopic carbon dioxide $\left({ }^{13} \mathrm{CO}_{2}\right)$ were measured with the Tm:fiber pumped OPO. Measurements of the absorption spectra were carried out by injecting a controlled amount of gas directly into the $\mathrm{N}_{2}$-purged Plexiglas enclosure, or by using an intra-cavity gas cell with length $48 \mathrm{~cm}$ and a volume of $\sim 30 \mathrm{~cm}^{3}$ (used for formaldehyde and carbon monoxide). The Brewster windows in the gas cell were made of 1-mm-thick $\mathrm{ZnS}$ that was chosen because of its comparatively low dispersion in the $2-5 \mu \mathrm{m}$ range (with zero-dispersion at $3.7 \mu \mathrm{m}$ ).

The main advantages with injecting gas directly into the OPO enclosure were simplic- 


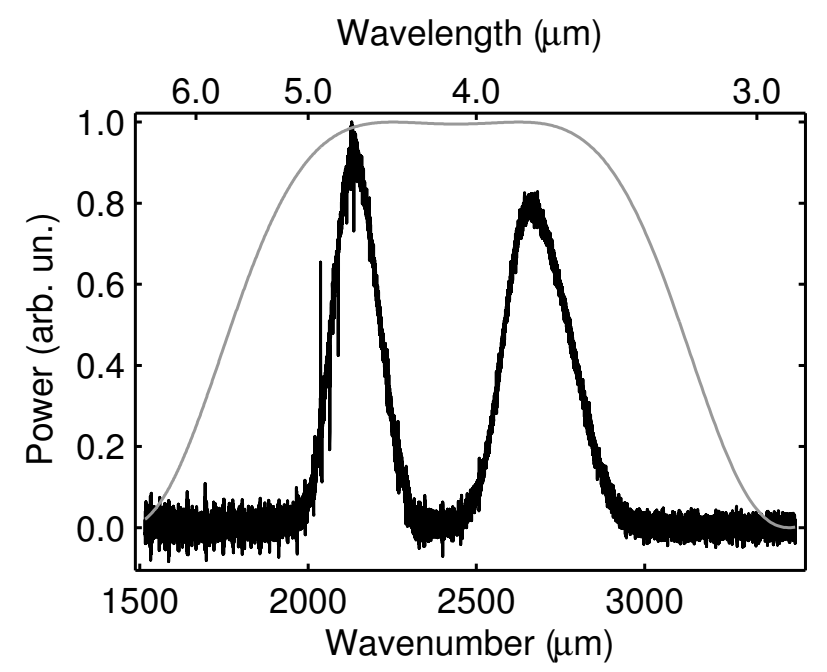

Fig. 8. Measured spectrum for one oscillation peak for the Tm:fiber-pumped OPO. Also shown (gray line) is the theoretical gain spectrum of the $500 \mu \mathrm{m}$ OP-GaAs crystal.

ity and the ability to exploit the entire cavity round-trip length as a physical path length through the gas. The disadvantages with this approach were the large volume of the Plexiglas enclosure (estimated to be 103 liter) and possible contamination of the optical surfaces of the OPO components. The advantages using the gas cell were the small volume of the cell, and the ability to easily control the flow rate and the pressure of the gas. The main disadvantage with the gas cell was the short cell length compared to the cavity round-trip distance $(\sim 0.5 \mathrm{~m}$ vs. $\sim 4 \mathrm{~m})$, but this could in principle be improved by using a different resonator geometry allowing longer intracavity gas cells.

All spectra were measured at 1 atm. pressure and a temperature of $21.5{ }^{\circ} \mathrm{C}$. The flowrate of gas through the intra-cavity cell was $0.2 \mathrm{l} / \mathrm{min}$. for formaldehyde, and a steadystate gas concentration was reached after $\sim 5 \mathrm{~min}$. of flowing. No flowing was used for carbon monoxide. Unless otherwise noted, the OPO output spectra were measured with a commercial (Nicolet 6700) FTIR spectrometer with a liquid $\mathrm{N}_{2}$-cooled $\mathrm{HgCdTe}$ detector. We used the maximum available resolution of $0.125 \mathrm{~cm}^{-1}$ of the FTIR instrument for most of the measurements. The total measurement time was in the range of 30 seconds up to 4 minutes, which includes averaging over 8 to 32 scans. First, the OPO was locked to an appropriate oscillation peak during measurements using the dither-and-lock technique. Then reference spectra were obtained by filling the OPO enclosure and gas cell, respectively, with $\mathrm{N}_{2}$ only. Finally, the trace gases were injected and the absorption spectra were determined from the ratio between these two measurements. The free-running pump lasers resulted in drifts of 
the OPO spectra on a time scale of the order of minutes, affecting the baseline in the relative OPO spectra. We therefore apply a baseline correction (determined by parts of the spectra between the absorption lines) of up to a few percent of the measured spectral intensity. In addition, an offset in wavenumber of up to $\sim 1 \mathrm{~cm}^{-1}$ was applied to the measured data, to compensate FTIR instrument errors and to match the positions of the measured absorption peaks to the HITRAN data.

\section{Results obtained with the Er-pumped system}

\section{A. Methane $\left(\mathrm{CH}_{4}\right)$}

A controlled amount of $0.2-1 \%$ methane in $\mathrm{N}_{2}$ was injected into the $\mathrm{N}_{2}$-flushed OPO enclosure. Based on the volume of the enclosure, the concentration of methane in the OPO cavity was estimated to be $8.5 \mathrm{ppm}$. Figure 9(a) shows measured and calculated intracavity

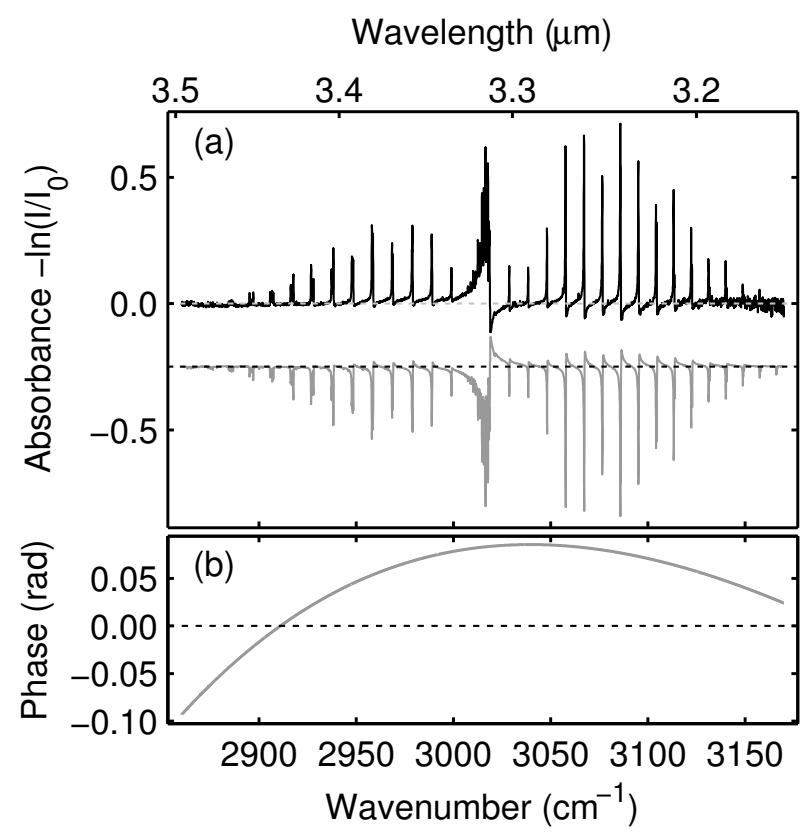

Fig. 9. (a) Measured (black) and calculated (gray) absorption spectra for $8.5 \mathrm{ppm}$ methane in nitrogen at 1 atm. pressure, corresponding to peak No. 4 in Fig. 6. The calculated spectrum is inverted and offset for clarity. (b) Phase shift $\Delta \phi(\nu)$, which was used for the calculation in (a).

spectra, corresponding to absorption of methane for the OPO using $0.8 \mathrm{~mm}$ of PPLN. The measurement time was $57 \mathrm{~s}$. The round-trip phase $\Delta \phi$ is determined from the calculated round-trip dispersion of the OPO cavity up to a linear frequency dependence. We adjust the linear part of $\Delta \phi$ to optimize the fit between the measured and the calculated spectrum. 
The calculated absorption of methane was based on the line intensities $S(\nu)$ and line halfwidths $\gamma$ from the HITRAN database [28]. The resulting complex susceptibility was used as an input to Eq. (8) together with the estimated round-trip phase $\Delta \phi$, which is shown in Fig. 9(b). We estimated the round-trip loss to be $25 \%$. We observe that the simulated spectrum reproduces the measured spectrum well. Figure 10 shows measured and calculated

\section{Wavelength $(\mu \mathrm{m})$}

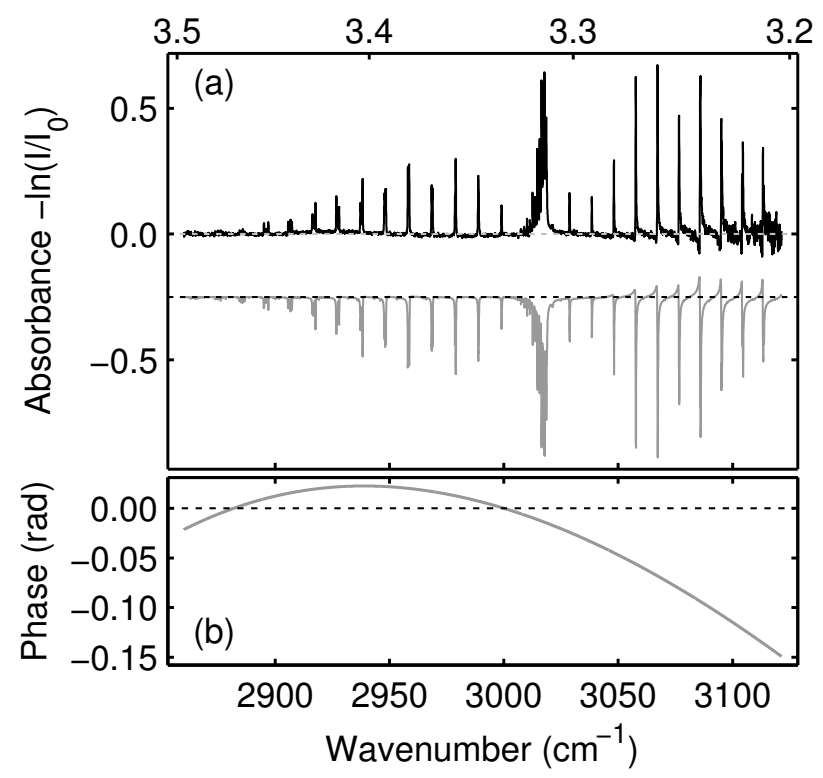

Fig. 10. (a) Measured (black) and calculated (gray) absorption spectra for $8.5 \mathrm{ppm}$ methane in nitrogen at $1 \mathrm{~atm}$. pressure for an adjacent oscillation peak (peak No. 5 in Fig. 6), compared to Fig. 9, The calculated spectrum is inverted and offset for clarity. (b) Phase shift $\Delta \phi(\nu)$, which was used for the calculation in (a).

spectra for an adjacent (in cavity length) OPO oscillation peak (No. 5), compared to Fig. 9. In the calculation, we assumed the required cavity length change between the two oscillation peaks to be $1.71 \mu \mathrm{m}$. Nominally, the change in cavity length between two adjacent peaks is equal to the pump wavelength [18], which is $1.56 \mu \mathrm{m}$. However, the observed spacing between the oscillation peaks varies within $\pm 15 \%$, which we attribute to intracavity dispersion.

Based on the measured absorption spectrum and the noise of the detector and the laser source, we estimated a detection limit of $1.7 \mathrm{ppb}$ for methane with the current experimental parameters. Compared to single peak detection, broadband spectroscopy detects multiple absorption peaks simultaneously, which leads to an increase of the sensitivity, as pointed out in Ref. [17]. For example, if there are $N$ equally strong absorption peaks, the multi- 
line advantage improves the detection limit by a factor $\sqrt{N}$. For methane, the sensitivity is improved by approximately one order of magnitude, depending on the spectral resolution [17]. In Sec. 8, we discuss the procedure of calculating the detection limit in our experiment.

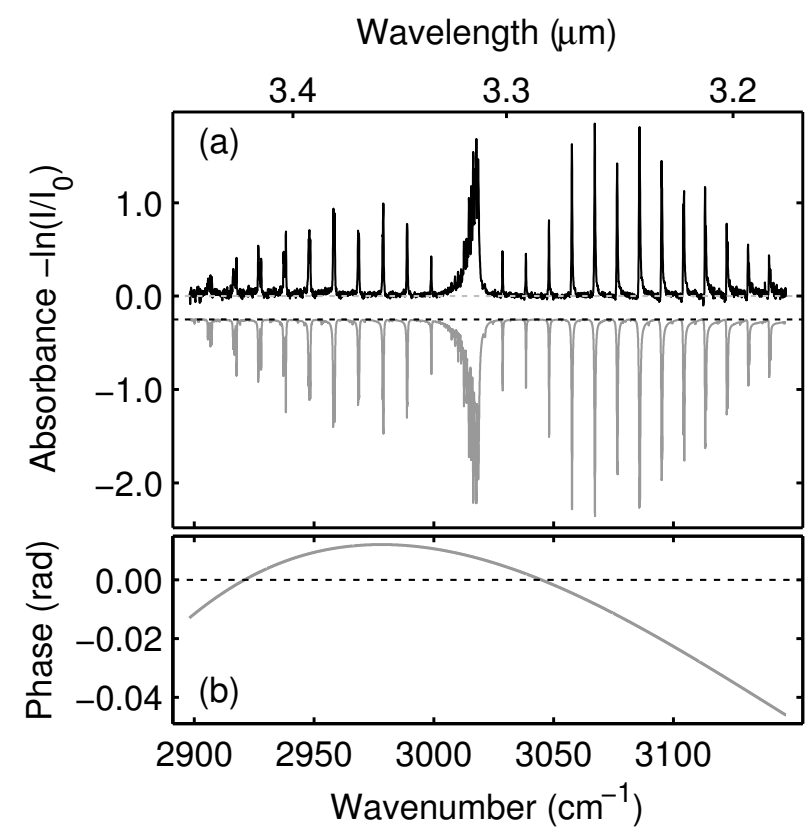

Fig. 11. (a) Measured (black) and calculated (gray) absorption spectra for $56 \mathrm{ppm}$ methane in nitrogen at $1 \mathrm{~atm}$. pressure. The calculated spectrum is inverted and offset for clarity. (b) Phase shift $\Delta \phi(\nu)$, which was used for the calculation in (a).

Figure 11 shows measured and calculated intracavity spectra for 56 ppm methane, with the Er:fiber-pumped OPO using $0.8 \mathrm{~mm}$ PPLN and close to zero intracavity dispersion. The estimated round trip loss is about $30 \%$, which is due to increased out-coupling, compared to Figs. (91) and (10). The spectrum was measured using a DTGS (deuterated triglycine sulfate) detector at room temperature, with 4 min. measurement time. As for Figs. (9) and (10), the linear part of the round-trip phase is adjusted to fit the calculated spectrum to the measured spectrum.

The concentration of methane in standard air is approximately $1.8 \mathrm{ppm}$, and many of the methane lines are overlapping with the absorption lines of water vapor. Figure 12 shows a part of the OPO spectrum with no $\mathrm{N}_{2}$-flushing of the cavity (relative humidity $33.6 \%$, temperature $21.5^{\circ} \mathrm{C}$ ). The selected part of the spectrum corresponds to a region where a strong absorption line of methane is not significantly interfering with absorption lines of water vapor. We clearly see the absorption feature of methane in air, which is compared in the same 


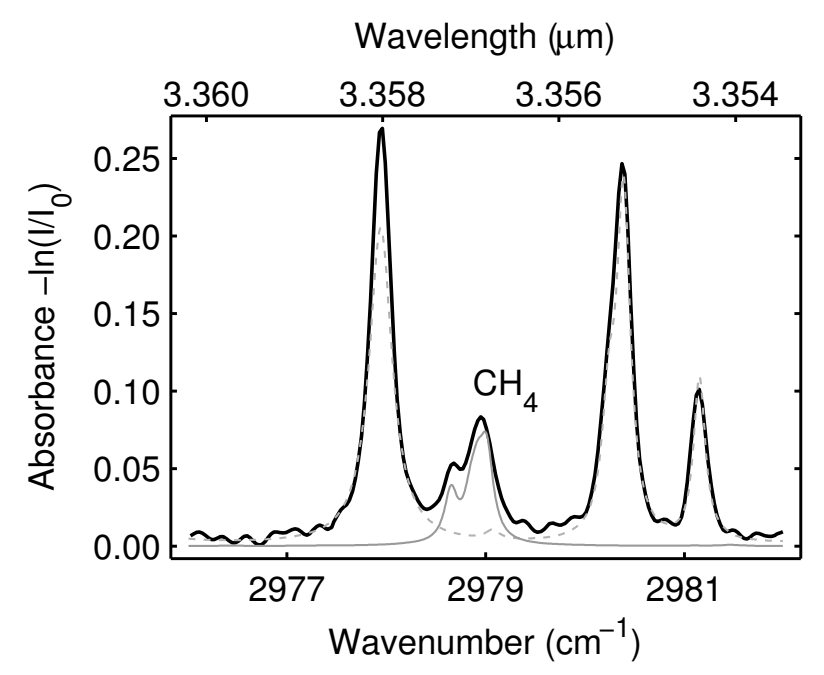

Fig. 12. Measured absorption spectrum for ambient air (black line) containing a methane peak. The HITRAN simulation for methane (solid gray line) and the three simulated (HITRAN) water vapor peaks (dashed gray line) are also shown. The effective path length is taken to be 8 times the round-trip length of the OPO cavity for the calculated spectra.

figure to a HITRAN simulation for $1.8 \mathrm{ppm}$ of methane and intracavity enhancement factor of 8. A reasonable match between experimental and calculated spectra is consistent with a round-trip loss of $25 \%$. Simulated water vapor peaks (HITRAN) for the above atmospheric conditions and enhancement factor are shown as dashed lines.

\section{B. Formaldehyde $\left(\mathrm{CH}_{2} \mathrm{O}\right)$}

Measurements of the absorption spectrum of formaldehyde were carried out using a gas cell with a continuous flow of $100 \mathrm{ppm}$ formaldehyde in $\mathrm{N}_{2}$. In these measurements we used a 500- $\mu$ m-long PPLN crystal to obtain a sufficiently wide spectrum, corresponding to peak 4 in Fig. 7(b), to cover the absorption region of formaldehyde from 3.3 to $3.7 \mu \mathrm{m}$. Figure 13(a) and (b) show measured and calculated spectra of formaldehyde when the gas cell is placed outside ( 2 min. measurement time) and inside (30 s measurement time) the OPO cavity, respectively. The absorption was enhanced by a factor of about 6 when the gas cell was placed inside the OPO cavity, giving a detection limit of approximately $0.31 \mathrm{ppm}$. This enhancement corresponds to an intracavity round-trip loss of $33 \%$ for the signal. This loss is higher than for the measurements with methane, but the increased round-trip loss may be due to a small misalignment of the gas cell.

For the fitted data we assumed a round-trip phase shift of $\Delta \phi=0$, because this gave a 


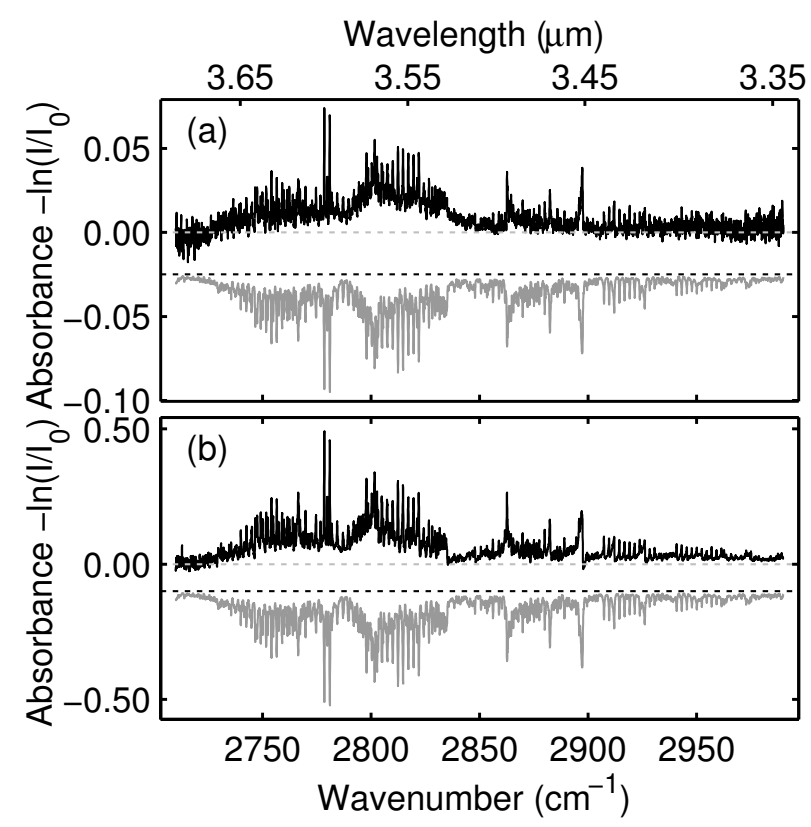

Fig. 13. Measured (black) and calculated absorption spectra (gray) for 100 ppm formaldehyde in nitrogen at $1 \mathrm{~atm}$. pressure. The calculated spectra are offset and shown on an inverted scale for clarity. (a) Extracavity spectra, (b) intracavity spectra. The effective path length is taken to be 6 times the length of the gas cell for the calculated spectrum in (b).

reasonable overlap with the measured data.

\section{C. Simultaneous measurement of acetylene $\left(\mathrm{C}_{2} \mathrm{H}_{2}\right)$ and methane $\left(\mathrm{CH}_{4}\right)$}

This experiment was performed to measure acetylene on one hand but also to show the capabilities of the intracavity OPO spectroscopy to detect more than one trace gas at once with reasonable high resolution. The use of a syringe allowed a precisely controlled injection of $400 \mathrm{ml}$ of $1000 \mathrm{ppm}$ acetylene in $\mathrm{N}_{2}$, followed by $15.0 \mathrm{ml}$ of $1 \%$ methane in $\mathrm{N}_{2}$, into the $\mathrm{N}_{2}$-flushed OPO enclosure, resulting in an estimated $3.8 \mathrm{ppm}$ acetylene concentration and $1.4 \mathrm{ppm}$ methane concentration, respectively. Figure 14(a) shows the measured reference spectrum (gray) and, underneath, the absorption intracavity spectrum (black), covering the absorption region of methane and acetylene, with the OPO operated with $0.8 \mathrm{~mm}$ of PPLN crystal length resonating at its peak No. 4. Figure 14(b) indicates the dispersive absorption spectrum of methane and the corresponding calculated spectrum. Figure 14(d) contains the detected absorption spectrum for acetylene which shows a similar amount of dispersion. Taking both traces simultaneously did not allow optimizing the spectrum for lowest dispersion 


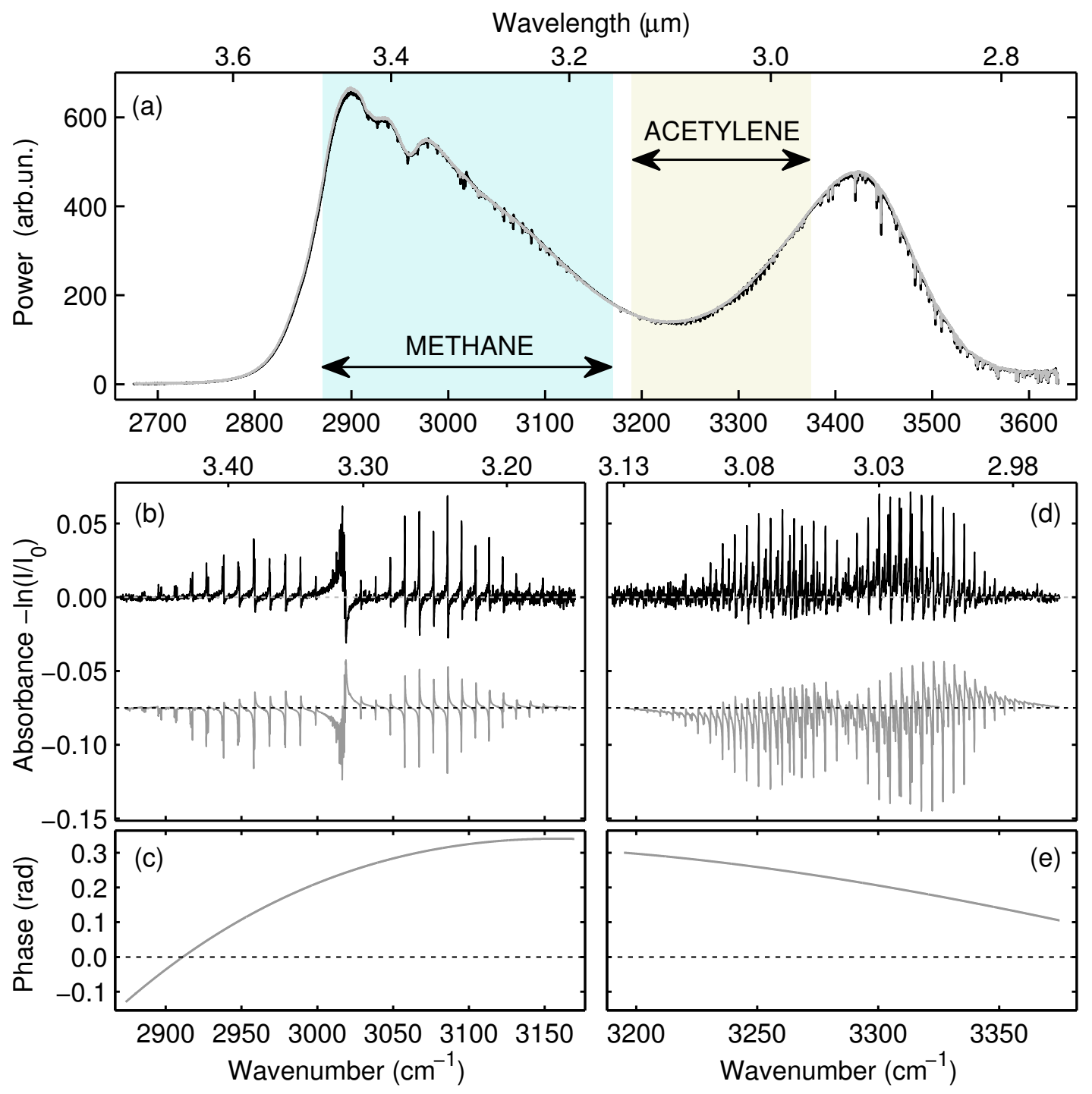

Fig. 14. (a) Showing reference intracavity spectrum (gray) and, underneath, the intracavity spectrum (black) with the absorption features present, while detecting methane and acetylene simultaneously inside the OPO. (b) Experimentally measured methane spectrum (black) at a concentration of $1.4 \mathrm{ppm}$ and the corresponding calculated spectrum (gray). (c) Round-trip phase shift for the calculated methane spectrum. (d) Experimentally measured acetylene spectrum (black) at a concentration of $3.8 \mathrm{ppm}$ and the corresponding calculated spectrum (gray). (e) Round-trip phase shift for the calculated acetylene spectrum. The calculated spectra in (b) and (d) are offset and shown on an inverted scale for clarity. 
across the relevant wavelength ranges while maintaining a good signal to noise ratio for both gases. Setting the FTIR to 17 scans per trace with a resolution of $0.125 \mathrm{~cm}^{-1}$ resulted in an acquisition time of $60 \mathrm{~s}$ for the reference as well as the absorption measurements. The simulated spectra were obtained using the phase shifts in Fig. 14(c) and (e) for methane and acetylene, respectively, while assuming a cavity round-trip loss of $25 \%$. We estimate a detection limit of $0.11 \mathrm{ppm}$ for acetylene with the current experimental conditions.

\section{D. Ethylene $\left(\mathrm{C}_{2} \mathrm{H}_{4}\right)$}

A carefully controlled injection of $5.2 \mathrm{l}$ of $1000 \mathrm{ppm}$ ethylene in $\mathrm{N}_{2}$ into the $\mathrm{N}_{2}$-flushed $\mathrm{OPO}$ enclosure resulted in an estimated ethylene concentration of $48 \mathrm{ppm}$ along the OPO path. Figure 15(a) provides the obtained absorption spectrum, resulting from the injected ethylene taken within $60 \mathrm{~s}$, with identical settings for the FTIR as described in Sec. 6.C. The

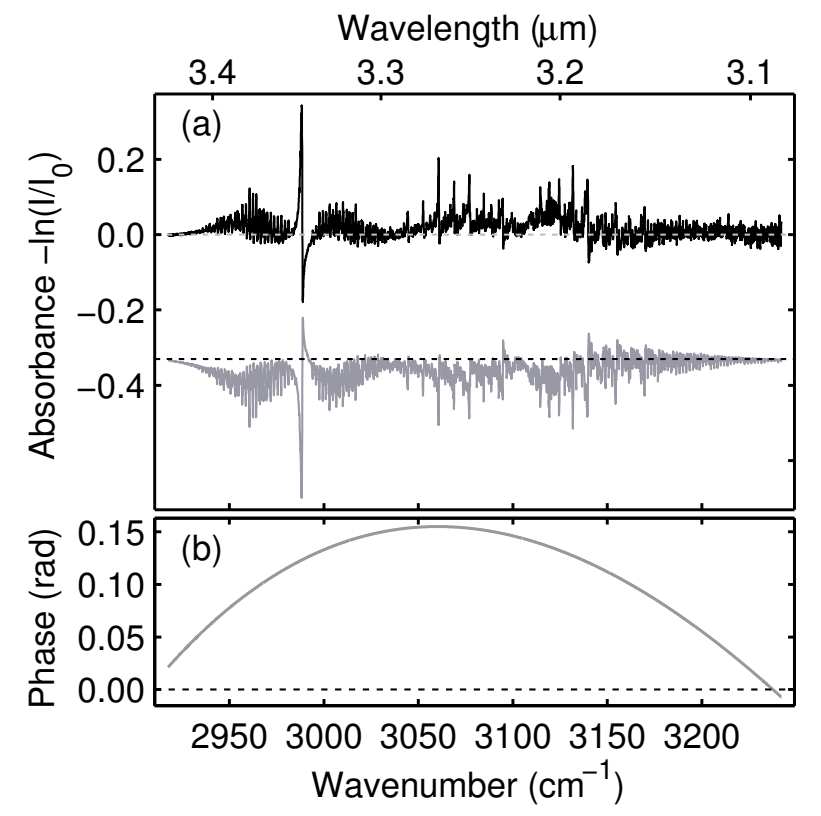

Fig. 15. (a) Experimentally measured ethylene spectrum (black) at a concentration of $48 \mathrm{ppm}$ and the corresponding calculated spectrum (gray). The calculated spectrum is inverted and offset for clarity. (b) Phase shift $\Delta \phi(\nu)$, which was used for the calculation in (a).

simulated spectrum was obtained using the phase shift shown in Fig. 15(b) and assuming a cavity round-trip loss of $25 \%$. We estimate a detection limit of $0.32 \mathrm{ppm}$ for ethylene with our setup. 


\section{Results with the Tm-pumped system}

The Tm-system represents an attractive system because of its wide bandwidth from 2.6$6.1 \mu \mathrm{m}$ at the $-30 \mathrm{~dB}$ level. Initial measurements of intracavity spectra of water vapor and isotopic $\mathrm{CO}_{2}$ in standard air have previously been presented in Refs. [19, 30] for this system.

\section{A. Carbon monoxide (CO)}

Carbon monoxide absorbs around $4.7 \mu \mathrm{m}$, and the Tm-system was tuned to this wavelength region by selecting an appropriate oscillation peak, similar to the one shown in Fig. 8 , The gas cell was filled with $50 \mathrm{ppm} \mathrm{CO}$ in He and placed inside the OPO cavity. Figure

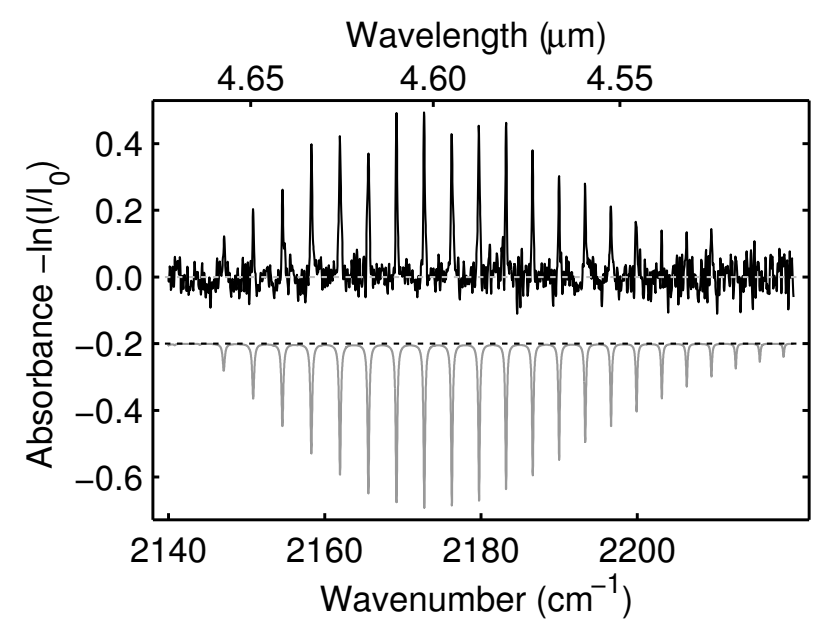

Fig. 16. Measured (black) and calculated (gray) absorption spectra for $50 \mathrm{ppm}$ carbon monoxide in helium at 1 atm. pressure. The calculated spectrum is offset and shown on an inverted scale for clarity. The effective path length is taken to be 7 times the length of the gas cell for the calculated spectrum.

16 shows the measured and calculated absorption spectrum of $\mathrm{CO}$, with a measurement time of $2 \mathrm{~min}$. In the calculations we assume a round-trip phase shift of $\Delta \phi=0$, which provided a reasonable fit to the measured data. The detection limit for CO is estimated to be $0.27 \mathrm{ppm}$. The increased noise properties of the Tm-system, compared to the Er-system, are attributed to instabilities (mode hopping) in the 790-nm laser diodes used for pumping the Tm:fiber amplifier. We observe that the intracavity absorption is enhanced by a factor of $\sim 7$, corresponding to a round-trip loss of approximately 30\%. However, this is higher than the expected round-trip loss of approximately $20 \%$, estimated in a previous publication of the Tm-system [19], and might be due to clipping of the signal beam introduced by the intracavity gas cell. For a perfectly aligned gas cell, the clipping is estimated to be a few 
percent.

\section{B. Isotopic carbon dioxide $\left({ }^{13} \mathrm{CO}_{2}\right)$}

Isotopic $\left({ }^{13} \mathrm{CO}_{2}\right)$ carbon dioxide is naturally present in the atmosphere at concentration of approximately $4 \mathrm{ppm}$ in volume [31], and can be easily detected using our Tm-laser based OPO without purging the cavity. The resolution of the spectrometer in this experiment was $0.5 \mathrm{~cm}^{-1}$ (we used model 80251 Oriel-Newport mid-IR spectrometer). Figure 17 shows

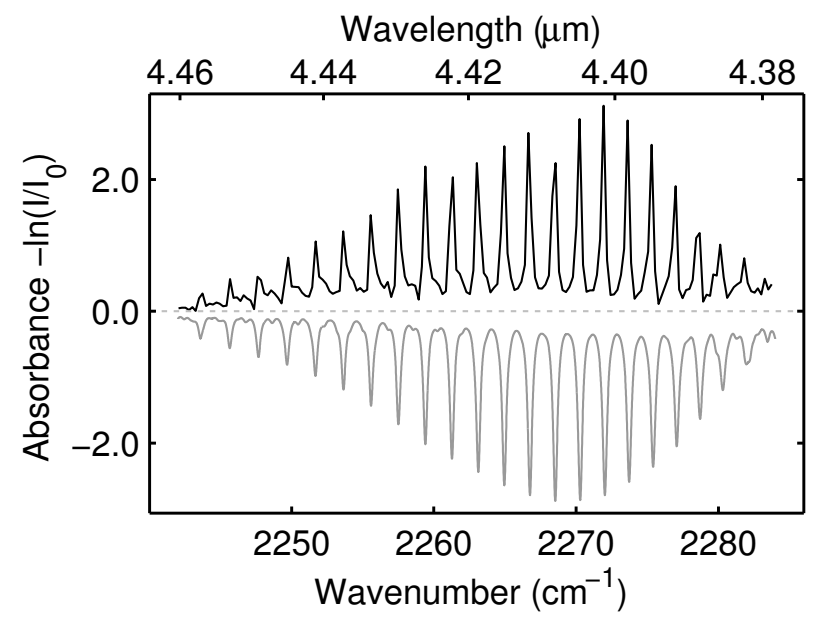

Fig. 17. Measured (black) and calculated (gray) molecular spectra of isotopic $\left({ }^{13} \mathrm{CO}_{2}\right)$ carbon dioxide. The simulation is based on the HITRAN database and is inverted for clarity.

measured and calculated (HITRAN, with matching spectral resolution of $0.5 \mathrm{~cm}^{-1}$ ) spectra of isotopic carbon dioxide. To match the two spectra in their peak values, we multiplied the HITRAN spectrum by 15 (assuming $\Delta \phi=0$ ), which means that our cavity absorption enhancement factor is larger than the one we typically observe. This discrepancy can be the result of an elevated (as compared to the average) amount of $\mathrm{CO}_{2}$ in the lab.

\section{Discussion}

The main motivation of using intracavity spectroscopy is to increase the effective path length through the gas, without the need of locking to an external Fabry-Perot cavity, or using an external multi-pass cell. The effective path length in our experiments dependents on the round-trip loss in the OPO cavity. We observe an absorption enhancement up to a factor of 8 , when the gas concentration was controlled, corresponding to a round-trip loss of $25 \%$. This loss is comparatively high, and could be due to clipping of the resonating beam in the OPO 
cavity. Based on the mirror and coating specifications, a cavity round-trip loss of $5-10 \%$ should be obtainable, resulting in an enhancement factor of $20-40$. Maximum effective path lengths are thus in the range $100-200 \mathrm{~m}$. We emphasize that once the cavity round-trip loss and dispersion has been determined (e.g. using a known gas with known concentration), both the enhancement factor and expected lineshapes are known for all molecules to be measured and thus absolute concentration measurements can be performed. Table 1 shows

Table 1. Estimated detection limits.

\begin{tabular}{ccccc}
\hline Gas name & $\begin{array}{c}\text { Det. limit } \\
{[\mathrm{ppm}]}\end{array}$ & $\begin{array}{c}\text { Meas. time } \\
{[\mathrm{s}]}\end{array}$ & $\begin{array}{c}\text { Phys. path length } \\
{[\mathrm{m}]}\end{array}$ & System \\
\hline Methane $\left(\mathrm{CH}_{4}\right)$ & 0.0017 & 57 & 3.75 & $\operatorname{Er}$ \\
Formaldehyde $\left(\mathrm{CH}_{2} \mathrm{O}\right)$ & 0.31 & 30 & 0.48 & $\operatorname{Er}$ \\
Acetylene $\left(\mathrm{C}_{2} \mathrm{H}_{2}\right)$ & 0.11 & 60 & 3.75 & Er \\
Ethylene $\left(\mathrm{C}_{2} \mathrm{H}_{4}\right)$ & 0.32 & 60 & 3.75 & $\operatorname{Er}$ \\
Carbon monoxide $(\mathrm{CO})$ & 0.27 & 120 & 0.48 & $\mathrm{Tm}$ \\
Isotopic carbon dioxide $\left({ }^{13} \mathrm{CO}_{2}\right)$ & 0.0024 & 90 & 4.00 & $\mathrm{Tm}$ \\
\hline
\end{tabular}

estimated detection limits for each of the six molecules investigated. For calculating the molecular detection limit in our experiment, we have used the following procedure: First, we obtain the experimental set of points for the intracavity molecular absorbance $S\left(\nu_{n}\right)$. Second, from the known cavity dispersion and HITRAN database, we obtain the theoretical absorbance spectrum $T\left(\nu_{n}\right)$ - for the same uniformly spaced set of points $\nu_{n}$. We remove the baseline from theoretical spectrum, since the baseline in our experiment is not well defined (we fit it to the valleys between absorption peaks). Next, we 'project' our experimental spectrum onto the theoretical one, that is we integrate the product $S(\nu) T(\nu)$ over frequency, such that $I_{1}=\sum S\left(\nu_{n}\right) T\left(\nu_{n}\right)$. Then we estimate a standard deviation $\sigma$ (noise) of our experimental spectrum and computer-generate random 'noise' $N\left(\nu_{n}\right)$ over the set $\nu_{n}$, with normal distribution and standard deviation $\sigma$ and project the noise onto the theoretical spectrum, i.e. integrate $N(\nu) T(\nu)$ over frequency and get $I_{2}=\sum N\left(\nu_{n}\right) T\left(\nu_{n}\right)$. The sum $I_{2}$ fluctuates around zero with each computer run, and by doing many ( $>1000)$ such runs, we get the standard deviation $\Delta I_{2}$. Finally, we calculate the minimal detectable concentration as the concentration used in the experiment, divided by a factor $I_{1} / \Delta I_{2}$ (which is an effective signal-to-noise factor). We think that sensitivity enhancement due to our 'matched filter' approach is totally consisted with the 'multiple peaks' advantage described in Ref. [17].

During our experiments, the pump laser was free-running, and we observed a drift of the 
OPO spectra on a time scale of the order of minutes. This resulted in baseline drifts in the measured absorption spectra. One improvement would be to lock the pump laser to an external frequency reference. The OPO output would then be stabilized due to the self phase-locking mechanism of degenerate OPOs [32].

\section{Conclusions}

By using synchronously pumped OPOs operating around degeneracy, we obtain ultrabroadband mid-IR radiation suitable for coherent spectroscopy in the Fourier domain. A large instantaneous bandwidth of up to $800 \mathrm{~cm}^{-1}$ allows detection of several trace gases simultaneously. Spectroscopic detection of six molecules in trace amounts has been performed in the wavelength range of $2.5-5 \mu \mathrm{m}$. By injecting the gases inside the OPO cavities we obtained substantial enhancement of the effective path length and achieved detection limits down to part-per-billion level in volume. Our intracavity sensing approach offers great simplicity and compactness, which might be a great asset for future applications. The dispersive spectral features that we observe at some circumstances are well reproduced using a simple model for propagation in a dispersive Fabry-Perot cavity. These features can be predicted a priori from knowledge of dispersion of the intracavity elements; on the other hand, if the dispersion of the individual components is not known, the overall dispersion can be precisely mapped by injecting trace amounts of known molecules and analyzing the shapes of spectral peaks. By decreasing the OPO loss and increasing the finesse of the OPO cavity we expect an improvement of detection limits, down to sub-ppb levels. This will also require better dispersion compensation, e.g. using chirped dielectric mirrors. Utilizing intracavity cells with low ( $\sim 0.1$ atm.) gas pressure will allow better specificity of molecular recognition because of sharper spectral features. With further development, this system may find important applications in trace gas detection and real time human breath analysis - with further enhancement possible through the use of dual-comb multi-heterodyne methods.

\section{Acknowledgments}

We greatly thank NASA, Office of Naval Research, Air Force Office of Scientific Research, Agilent Technologies, Sanofi-Aventis, Stanford University Bio-X, Stanford Medical School

and Stanford Woods Institute for their financial support. We also thank Leo Hollberg, Robert Byer, Martin Fejer and Alex Kachanov for the most useful discussions. M.W.H. is indebted to Gunnar Arisholm for his comments on the manuscript.

\section{References}

1. M. B. Esler, D. W. T. Griffith, S. R. Wilson, and L. P. Steele, "Precision trace gas analysis by ft-ir spectroscopy. 1. Simultaneous analysis of $\mathrm{CO}_{2}, \mathrm{CH}_{4}, \mathrm{~N}_{2} \mathrm{O}$, and $\mathrm{CO}$ in 
air," Anal. Chem. 72, 206-215 (2000).

2. A. Schliesser, M. Brehm, F. Keilmann, and D. W. van der Weide, "Frequency-comb infrared spectrometer for rapid, remote chemical sensing," Opt. Express 13, 9029-9038 (2005).

3. M. J. Thorpe, D. Balslev-Clausen, M. S. Kirchner, and J. Ye, "Cavity-enhanced optical frequency comb spectroscopy: application to human breath analysis," Opt. Express 16, $2387-2397$ (2008).

4. D. D. Arslanov, K. Swinkels, S. M. Cristescu, and F. J. M. Harren, "Real-time, subsecond, multicomponent breath analysis by optical parametric oscillator based off-axis integrated cavity output spectroscopy," Opt. Express 19, 24078-24089 (2011).

5. T. H. Risby and F. K. Tittel, "Current status of midinfrared quantum and interband cascade lasers for clinical breath analysis," Opt. Eng. 49, 111123 (2010).

6. S. A. Diddams, "The evolving optical frequency comb," J. Opt. Soc. Am. B 27, B51-B62 (2010).

7. S. A. Diddams, L. Hollberg, and V. Mbele, "Molecular fingerprinting with the resolved modes of a femtosecond laser frequency comb," Nature 445, 627-630 (2007).

8. E. Sorokin, I. T. Sorokina, J. Mandon, G. Guelachvili, and N. Picqué, "Sensitive multiplex spectroscopy in the molecular fingerprint $2.4 \mu \mathrm{m}$ region with a $\mathrm{Cr}^{2+}: \mathrm{ZnSe}$ femtosecond laser," Opt. Express 15, 16540-16545 (2007).

9. J. Mandon, G. Guelachvili, and N. Picqué, "Fourier transform spectroscopy with a laser frequency comb," Nature Photonics 3, 99-102 (2009).

10. F. Keilmann, C. Gohle, and R. Holzwarth, "Time-domain mid-infrared frequency-comb spectrometer," Opt. Lett. 29, 1542-1544 (2004).

11. M. J. Thorpe and J. Ye, "Cavity-enhanced direct frequency comb spectroscopy," Appl. Phys. B 91, 397-414 (2008).

12. B. Bernhardt, A. Ozawa, P. Jacquet, M. Jacquey, Y. Kobayashi, T. Udem, R. Holzwarth, G. Guelachvili, T. W. Hänsch, and N. Picque, "Cavity-enhanced dual-comb spectroscopy," Nature Phot. 4, 55-57 (2009).

13. X. D. D. Vaernewijck, K. Didriche, C. Lauzin, A. Rizopoulos, M. Herman, and S. Kassi, "Cavity enhanced ftir spectroscopy using femto opo absorption source," Mol. Phys. 109, 2173-2179 (2011).

14. A. Foltynowicz, P. Masłowski, A. Fleisher, B. Bjork, and J. Ye, "Cavity-enhanced optical frequency comb spectroscopy in the mid-infrared application to trace detection of hydrogen peroxide," Applied Physics B pp. 1-13 (2012).

15. K. A. Tillman, R. R. J. Maier, D. T. Reid, and E. D. McNaghten, "Mid-infrared absorption spectroscopy of methane using a broadband femtosecond optical parametric oscillator based on aperiodically poled lithium niobate," J. Opt. A: Pure Appl. Opt. 7, 
S408-S414 (2005).

16. F. Adler, K. C. Cossel, M. J. Thorpe, I. Hartl, M. E. Fermann, and J. Ye, "Phasestabilized, 1.5 W frequency comb at 2.8-4.8 $\mu \mathrm{m}$," Opt. Lett. 34, 1330-1332 (2009).

17. F. Adler, P. Masłowski, A. Foltynowicz, K. C. Cossel, T. C. Briles, I. Hartl, and J. Ye, "Mid-infrared Fourier transform spectroscopy with a broadband frequency comb," Opt. Express 18, 21861-21872 (2010).

18. N. Leindecker, A. Marandi, R. L. Byer, and K. L. Vodopyanov, "Broadband degenerate opo for mid-infrared frequency comb generation," Opt. Express 19, 6296-6302 (2011).

19. N. Leindecker, A. Marandi, R. L. Byer, K. L. Vodopyanov, J. Jiang, I. Hartl, M. Fermann, and P. G. Schunemann, "Octave-spanning ultrafast opo with 2.6-6.1 $\mu \mathrm{m}$ instantaneous bandwidth pumped by femtosecond Tm-fiber laser," Opt. Express 20, 7046-7053 (2012).

20. V. M. Baev, T. Latz, and P. E. Toschek, "Laser intracavity absorption spectroscopy," Appl. Phys. B 69, 171-202 (1999).

21. W. Brunner and H. Paul, "The optical parametric oscillator as a means for intracavity absorption spectroscopy," Opt. Comm. 19, 253-256 (1976).

22. K.-J. Boller and T. Schröder, "Demonstration of broadband intracavity spectroscopy in a pulsed optical parametric oscillator made of $\beta$-barium borate," J. Opt. Soc. Am. B 10, 1778-1784 (1993).

23. A. Foltynowicz, T. Ban, P. Masłowski, F. Adler, and J. Ye, "Quantum-noise-limited optical frequency comb spectroscopy," Phys. Rev. Lett. 107, 233002 (2011).

24. K. L. Vodopyanov, E. Sorokin, I. T. Sorokina, and P. G. Schunemann, "Mid-ir frequency comb source spanning 4.4-5.4 $\mu \mathrm{m}$ based on subharmonic GaAs optical parametric oscillator," Opt. Lett 36, 2275-2277 (2011).

25. V. L. Kalashnikov and E. Sorokin, "Soliton absorption spectroscopy," Phys. Rev. A 81, $033840(2010)$.

26. A. Marandi, N. C. Leindecker, V. Pervak, R. L. Byer, and K. L. Vodopyanov, "Coherence properties of a broadband femtosecond mid-ir optical parametric oscillator operating at degeneracy," Opt. Express 20, 7255-7262 (2012).

27. W. Demtröder, Laser Spectroscopy - Basic Concepts and Instrumentation (Springer, 2003).

28. "The HITRAN database," http://www.cfa.harvard.edu/HITRAN/.

29. L. Gianfrani, R. W. Fox, and L. Hollberg, "Cavity-enhanced absorption spectroscopy of molecular oxygen," J. Opt. Soc. B 16, 2347-2254 (1999).

30. M. W. Haakestad, N. Leindecker, A. Marandi, J. Jiang, I. Hartl, M. Fermann, and K. L. Vodopyanov, "Broadband intracavity molecular spectroscopy with a degenerate mid-IR OPO," in "Conference on Lasers and Electro-Optics (CLEO)," (San Jose, California, USA, 2012). Paper no. CF2C.2. 
31. "Wikipedia," http://en.wikipedia.org/.

32. S. T. Wong, K. L. Vodopyanov, and R. L. Byer, "Self-phase-locked divide-by-2 optical parametric oscillator as a broadband frequency comb source," J. Opt. Soc. Am. B 27, 876-882 (2010). 